\title{
Impact of COVID-19 Pandemic on Rheumatology Practice in Latin America
}

\author{
Daniel G. Fernández-Ávila ${ }^{1}$ iD, Julián Barahona-Correa ${ }^{2}$, Diana Romero-Alvernia ${ }^{2}$, Sergio Kowalski ${ }^{3}$, \\ Ana Sapag ${ }^{4}$, Antonio Cachafeiro-Vilar ${ }^{5}$, Belia Meléndez ${ }^{6}$, Carlos Santiago-Pastelín ${ }^{7}$, Daniel Palleiro ${ }^{8}$ iD, \\ Dina Arrieta ${ }^{9}$, Gil Reyes ${ }^{10}$, Guillermo J. Pons-Estel ${ }^{11}$ (D), Jossiell Then-Báez ${ }^{12}$, Manuel F. Ugarte-Gil ${ }^{13}$ (D), \\ Mario H. Cardiel ${ }^{14}$, Nelly Colman ${ }^{15}$, Nilmo Chávez ${ }^{16}$, Paula I. Burgos ${ }^{17}$, Rubén Montúfar ${ }^{18}$, \\ Sayonara Sandino ${ }^{19}$, Yurilis J. Fuentes-Silva ${ }^{20}$ iD, and Enrique R. Soriano ${ }^{21}$ iD
}

ABSTRACT. Objective. To describe the effect of the coronavirus disease 2019 (COVID-19) pandemic on Latin American rheumatologists from a professional, economic, and occupational point of view.

Methods. We conducted an observational cross-sectional study using an online survey sent to rheumatologists of each non-English-speaking country member of the Pan American League of Rheumatology Associations (PANLAR). A specific questionnaire was developed.

Results. Our survey included 1097 rheumatologists from 19 Latin American countries. Median (IQR) age of respondents was 48 (40-59) years and 618 (56.3\%) were female. Duration of practice since graduation as a rheumatologist was 17 years, and 585 (53.3\%) were aged < 50 years. Most rheumatologists worked in private practice $(81.8 \%)$ and almost half worked in institutional outpatient centers $(55 \%)$ and inpatient care (49.9\%). The median number of weekly hours (IQR) of face-to-face practice before the pandemic was 27 (15-40) hours, but was reduced to 10 (5-20) hours during the pandemic. Telehealth was used by 866 (78.9\%) respondents during the pandemic. Most common methods of communication were video calls (555; $50.6 \%)$, telephone calls $(499 ; 45.5 \%)$, and WhatsApp voice calls $(423 ; 38.6 \%)$. A reduction in monthly wages was reported by $946(86.2 \%)$ respondents. Consultation fees also were reduced and 88 (8\%) rheumatologists stated they had lost their jobs. A reduction in patient adherence to medication was reported by nearly $50 \%$ of respondents. Eighty-one (7.4\%) rheumatologists received a COVID-19 diagnosis and 7 (8.6\%) of them were hospitalized.

Conclusion. The COVID-19 pandemic has reshaped rheumatology practice in Latin America and has had a profound effect on rheumatologists' behaviors and clinical practice.

Key Indexing Terms: COVID-19, guidelines, practice, rheumatology, telehealth

This study was supported by an unrestricted grant from the Pan American League of Rheumatology Associations (PANLAR).

${ }^{I}$ D.G. Fernández-Avila, MD, Unidad de Reumatología, Pontificia Universidad Javeriana - Hospital Universitario San Ignacio, Bogotá, Colombia; ${ }^{2} J$. Barahona-Correa, MD, D. Romero-Alvernia, MD, Departamento de Medicina Interna, Pontificia Universidad Javeriana - Hospital Universitario San Ignacio, Colombia; ${ }^{3}$ S. Kowalski, MD, Universidade Federal do Paraná, Paraná, Brazil; ${ }^{4}$ A. Sapag, MD, Hospital Universitario Japonés, Santa Cruz, Bolivia; ${ }^{5}$ A. Cachafeiro-Vilar, MD, Pacífica Salud - Hospital Punta Pacífica, Panama City, Panama; ${ }^{6}$ B. Meléndez, MD, Hospital de la Policía Nacional N1 Quito, Quito, Ecuador; ${ }^{7}$ C. Santiago-Pastelin, MD, Instituto Hondureño de la Seguridad Social, Tegucigalpa, Honduras; ${ }^{8} D$. Palleiro, MD, Instituto Nacional de Reumatología del Uruguay, Universidad de la República, Montevideo, Uruguay; ${ }^{9}$ D. Arrieta, MD, Hospital México, San José de Costa Rica, Costa Rica; ${ }^{10}$ G. Reyes, MD, Universidad de Ciencias Médicas de la Habana, Havana, Cuba; ${ }^{11}$ G.J. Pons-Estel, MD, Centro Regional de Enfermedades Autoinmunes y Reumáticas CREAR, Rosario, Argentina; ${ }^{12} \mathrm{~J}$. Then-Báez, $M D$, Hospital Metropolitano de Santiago (HOMS), Santiago, Dominican Republic; ${ }^{13}$ M.F. Ugarte-Gil, MD, Hospital Nacional Guillermo

\begin{abstract}
Almenara Irigoyen, EsSalud - Universidad Cientifica del Sur, Lima, Peru; ${ }^{14}$ M.H. Cardiel, MD, Centro de Investigación Clínica de Morelia, Morelia, Mexico; ${ }^{15}$ N. Colman, MD, Hospital de Clínicas - Universidad Nacional de Asunción, Asunción, Paraguay; ${ }^{16} \mathrm{~N}$. Chávez, MD, Instituto Guatemalteco de Seguridad Social - Universidad San Carlos de Guatemala, Ciudad de Guatemala, Guatemala; ${ }^{17}$ P.I. Burgos, MD, Pontificia Universidad Católica de Chile, Santiago de Chile, Chile, ${ }^{18} R$. Montúfar, MD, Consultorio de Especialidades del Instituto Salvadoreño del Seguro Social, San Salvador, El Salvador; ${ }^{19}$ S. Sandino, MD, Instituto Médico Ganna, Managua, Nicaragua; ${ }^{20}$ Y.J. Fuentes-Silva, MD, Centro Clínico Universitario de Oriente - Universidad de Oriente, Ciudad Bolivar, Venezuela; ${ }^{21}$ E.R. Soriano, $M D$, Sección Reumatología, Servicio de Clinica Médica Hospital Italiano de Buenos Aires - Instituto Universitario Hospital Italiano de Buenos Aires, Buenos Aires, Argentina.

The authors declare no conflicts of interest relevant to this article. Address correspondence to Dr. D.G. Fernández-Ávila, Cra 7 No. 40-62. Piso 7, Unidad de Reumatología, Hospital Universitario San Ignacio, Bogotá, Colombia.Email: daniel.fernandez@javeriana.edu.co.

Accepted for publication May 28, 2021.
\end{abstract}


The coronavirus disease 2019 (COVID-19) is caused by the severe acute respiratory syndrome coronavirus 2 (SARS-CoV-2) virus. ${ }^{1}$ The disease broke out in Wuhan, China, in December 2019, and was declared a pandemic and a major global health threat by the World Health Organization in March 2020.,3

Globally adopted preventive measures focused on mitigating infection risk and the impact of COVID-19. These measures focus primarily on social distancing, hand hygiene, and wearing a mask in public settings. Social distancing emerged as a main strategy in public health aimed at preventing SARS-CoV-2 dissemination, which had implications for the management of rheumatology patients. Strategies to reduce physician-patient encounters in the outpatient setting were implemented as a means to prevent the spread of COVID-19 and to protect both patients and healthcare providers. ${ }^{4,5,6}$

Patients with rheumatic diseases are usually under chronic pharmacological immunosuppression, which could make them more susceptible to infections. There is biological plausibility to consider these patients at "high risk" for SARS-CoV-2 infection and development of COVID-19. These vulnerable populations face a dilemma between potential exposure to the virus and the need for medical care. Therefore, a change in the behavioral patterns of rheumatic patients and of rheumatologists was expected. $3,7,8,9$

Infectious outbreaks that require a change in daily habits are not new. These behavioral patterns are mediated by perceived susceptibility, perceived severity, and perceived benefits, barriers, and signals that elicit an impulse to take action. ${ }^{10}$ Experience from previous outbreaks has shown in the general population and in healthcare workers a favorable tendency to comply with hygiene instructions and social isolation. However, changes in daily habits, the adoption of preventive measures, and the economic uncertainty associated with quarantines are frequently reported. ${ }^{11-19}$

In addition to disruptions due to frequent lockdowns, quarantine, and the social distancing constraints, rheumatology clinical practice faced additional challenges. There are some studies about a change of habits and behaviors in healthcare workers during a pandemic. ${ }^{5,6,13,19}$ Management of outpatients has been described as a potentially difficult issue due to the lack of preparedness and disaster planning that could be effective in these situations. ${ }^{20}$

Studies about attitudes and behaviors are used to research the response and behavior patterns in communities facing the development and prevention of a new disease. ${ }^{18,19,20}$ Considering that rheumatic patients are a potentially vulnerable population, changes in behavioral patterns are expected in both patients and their physicians. This situation has not been previously explored during the current COVID-19 pandemic in Latin American countries.

Therefore, we conducted a survey to explore behaviors, attitudes, and changes in the practice of rheumatology during the COVID-19 pandemic in non-English-speaking countries of Latin America. The survey included both rheumatic patients and rheumatologists; however, in this article, only results from physicians will be presented.

\section{METHODS}

Objective. The objective of our study was to describe attitudes and practices of Latin American rheumatologists related to the management and follow-up of their patients during the COVID-19 pandemic. Our specific goal was to describe general demographic characteristics of physicians, and the way that the COVID-19 pandemic affected rheumatologists from a professional, economic, and occupational point of view.

Study design. We conducted an observational cross-sectional study using an online survey sent to rheumatologists of each non-English-speaking country with Pan American League of Rheumatology Associations (PANLAR) membership.

Sample. Convenience sampling was used, so the sample size was not calculated. A 30-day window was established for data collection throughout June and July 2020

Inclusion and exclusion criteria. Rheumatologists from PANLAR-affiliated countries with the ability and desire to complete the survey were included. Rheumatologists who currently did not have clinical practice for patients were excluded.

Data collection and instruments. The link to the survey was sent to the leading rheumatologist of each country using the REDCap (Research Electronic Data Capture) platform. This rheumatologist was responsible for disseminating the survey among colleagues in their country through the local rheumatology association. The survey was developed in Spanish and later translated to Portuguese by a Brazilian researcher (SK). The REDCap platform was used for data storage.

A specific instrument was developed based on previous experiences $^{12-15,17-19}$ to assess demographic and clinical information, self-reported disease, use of medications, symptoms suggestive of COVID-19, confirmed diagnosis of COVID-19, and request for medical consultation or hospitalization. Since data were self-reported, researchers did not have access to confirmatory evidence. The obtained information was anonymous.

The following subjects were evaluated using a set of answer options such as yes, no, don't know/no answer, a Likert scale, or answers with specific values (for example, questions about income or the number of hours spent consulting):

- Attitudes. Questions evaluated the degree of agreement with the recommendations for individual and social care, perceived susceptibility, and the ease of communication through digital and remote means. The willingness to adopt alternatives to in-person medical care and the perceived importance of crisis management were evaluated.

- Practices. Physicians were asked about behaviors and alternatives to guarantee the continuity of medical care, biosafety procedures, participation in multidisciplinary groups involved in designing institutional guidelines, strategies for patient management, and the need to offer medical service outside the subspecialty area.

An initial pilot survey was carried out with rheumatic patients and rheumatologists in Bogotá, Colombia, to confirm an adequate understanding of the questions and to define the period of time required for patients and rheumatologists to complete the survey. Its applicability was evaluated by digital means. The survey was tweaked according to identified difficulties before the generalized distribution of the instrument.

Statistical analysis. Descriptive statistics were performed by calculating measures of central tendency for quantitative variables and using counts and percentages for qualitative and nominal variables.

Ethics. This study is governed by the ethical principles of the Declaration of Helsinki and according to the scientific, technical, and administrative regulations for health research stated by Resolution 8430 of 1993 of the Colombian Health Ministry. By the same resolution, the study is considered a risk-free investigation. Confidentiality of the data was maintained through the use of secure databases. The study was approved by the Research and Ethics Committee of the Hospital Universitario San Ignacio and the Pontificia Universidad Javeriana (approval 2020/106). 


\section{RESULTS}

Our study included 1097 rheumatologists from 19 Latin American countries. From these, 1052 (96\%) managed adult patients while $45(4 \%)$ were pediatric rheumatologists. The countries that contributed the largest number of rheumatologists were Brazil (276, 25.2\%), Mexico (229, 20.9\%), Colombia (140, 12.8\%), and Argentina (120, 10.9\%). Table 1 shows the number and percentage of respondents per country.

The median age of respondents was 48 (IQR 40-59) years. There were 618 (56.3\%) female respondents and the median duration of practice since graduating as a rheumatologist was 17 (IQR 7-28) years. Out of the total sample, 585 respondents $(53.3 \%)$ were aged $<50$ years and approximately two-thirds of respondents $(n=730,66.5 \%)$ reported $>10$ years of experience.

Most rheumatologists worked in private practice (897 respondents; $81.8 \%)$, whereas $603(55.0 \%)$ worked in institutional outpatient centers and 547 (49.9\%) in inpatient care. Most practice time was spent in private practice (50 [IQR 30-90] h/week) and institutional outpatient centers (45 [IQR 30-65] h/week,). Other practice scenarios and practice time distribution can be seen in Table 2. Of note, the respondents could work in more than 1 environment of care delivery.

Eighty-one (7.4\%) rheumatologists received a COVID-19 diagnosis. Of these, 7 (8.6\%) of these 81 rheumatologists were hospitalized for a median of 11 (IQR 5-12) days. None of them required mechanical ventilation. Pharmacological treatment varied (Table 3 ).

The median number of hours of in-person practice before the pandemic was 27 (IQR 15-40), whereas the median number of patients seen face-to-face per hour was 3 (2-4). During the pandemic, 598 (54.5\%) rheumatologists continued offering in-person care, but the median number of hours of in-person

Table 1. Rheumatologists per country in Latin America who responded to the PANLAR survey $(\mathrm{n}=1097)$.

\begin{tabular}{lc}
\hline Country & $\mathrm{n}(\%)$ \\
\hline Argentina & $120(10.9)$ \\
Bolivia & $10(0.9)$ \\
Brazil & $276(25.2)$ \\
Chile & $23(2.1)$ \\
Colombia & $140(12.8)$ \\
Costa Rica & $12(1.1)$ \\
Cuba & $15(1.4)$ \\
Dominican Republic & $15(1.4)$ \\
Ecuador & $37(3.4)$ \\
El Salvador & $20(1.8)$ \\
Guatemala & $18(1.6)$ \\
Honduras & $9(0.8)$ \\
Mexico & $229(20.9)$ \\
Nicaragua & $12(1.1)$ \\
Panama & $16(1.5)$ \\
Paraguay & $31(2.8)$ \\
Peru & $30(2.7)$ \\
Uruguay & $26(2.4)$ \\
Venezuela & $58(5.3)$ \\
\hline
\end{tabular}

PANLAR: Pan American League of Rheumatology Associations.
Table 2. Practice scenarios and practice time distribution.

Respondents, n (\%) Time distribution,
h/week (IQR)

\begin{tabular}{lcc}
\hline Private practice & $897(81.8)$ & $50(30-90)$ \\
Institutional outpatient center & $603(55.0)$ & $45(30-65)$ \\
Inpatient care & $547(49.9)$ & $15(10-30)$ \\
Teaching & $430(39.2)$ & $15(10-23.6)$ \\
Research & $276(25.2)$ & $15(10-20.8)$ \\
Pharmaceutical industry & $152(13.9)$ & $5(5-10)$ \\
Other & $46(4.2)$ & $50(14.2-100)$ \\
\hline
\end{tabular}

Table 3. Pharmacological treatment received by rheumatologists with a diagnosis of COVID-19 $(\mathrm{n}=81)$.

\begin{tabular}{lc}
\hline Medication & $\mathrm{n}(\%)$ \\
\hline Hydroxychloroquine & $44(54.3)$ \\
Chloroquine & $2(2.5)$ \\
Lopinavir/ritonavir & $2(2.5)$ \\
Azithromycin & $45(55.6)$ \\
Amoxicillin & $9(11.1)$ \\
Colchicine & $4(5)$ \\
Other & $42(51.8)$ \\
None & $17(21)$ \\
\hline
\end{tabular}

COVID-19: coronavirus disease 2019.

practice was reduced to 10 (IQR 5-20) and the median number of patients seen face-to-face per hour was 2 (IQR 1-2.1).

Telehealth was used by 866 (78.9\%) respondents during the pandemic. The most commonly used methods of communication were telephone calls $(499,45.5 \%)$ and WhatsApp voice calls $(423,38.6 \%)$. Communication methods can be seen in Table 4.

The telehealth option was not offered by 231 (21.1\%) of the respondents. Among other reasons, respondents reported that they considered telehealth as an inadequate alternative for patients and that there was a lack of clarity regarding payment methods (Table 5).

The median number of hours of telehealth care per week during the pandemic was 5 (IQR 2-10), whereas the median number of patients virtually treated by the hour was 2 (IQR $1-3$ ).

Table 4. Methods of telehealth communication used during the pandemic $(\mathrm{n}=1097)$.

\section{n (\%)}

\begin{tabular}{lc}
\hline Use of telehealth & $866(78.9)$ \\
Voice calls & \\
Telephone & $499(45.5)$ \\
Skype & $33(3)$ \\
WhatsApp & $423(38.6)$ \\
Video calls & \\
Skype & $71(6.5)$ \\
WhatsApp & $397(36.2)$ \\
Microsoft Teams & $87(7.9)$ \\
Other & $217(19.8)$
\end{tabular}


Table 5. Reasons reported by rheumatologists for not providing telehealth options to their patients $(\mathrm{n}=231,21.1 \%)$.

$\mathrm{n}(\%)$

Do not know how to use telehealth alternatives

$33(14.3)$

Do not have means to offer these alternatives

$25(10.8)$

Lack of clarity regarding payment methods

$60(26)$

Do not consider telehealth alternatives as adequate

for their patients

$100(43)$

Patients did not accept these alternatives

$27(11.7)$

Continued in-person visits

$92(39.8)$

Other reasons

$41(17.7)$

The types of rheumatology virtual visits delivered by respondents were first-time visits $(8,0.9 \%)$, follow-up visits ( 478 , $55.2 \%)$, and both types of visits (380, 43.9\%).

Aside from face-to-face medical visits, rheumatologists reported using other communication channels with their patients, such as WhatsApp, phone calls, and email (Table 6).

The number of rheumatologists who agreed that telehealth was a valid strategy during the pandemic was 940 (85.7\%), but only $546(49.8 \%)$ believed that telehealth would hypothetically continue to be a valid option after the end of the pandemic.

The economic and occupational impact evaluation showed that $946(86.2 \%)$ respondents reported a reduction in monthly wages. The percentage reduction in monthly wages was $50 \%$ (IQR 30-60). Respondents reported 70\% (IQR 50-95\%) of appointments were cancelled, and 88 (8\%) rheumatologists stated they had lost their jobs. Four hundred (46.2\%) respondents reported a reduction of consultation fees; the percentage reduction from the baseline fee was $42.9 \%$ (IQR 25-0).

Results showed a reduction in physician-assessed patient adherence to medication as reported by $504(45.9 \%)$ rheumatologists in patients receiving synthetic drugs and by 482 (43.9\%) rheumatologists in patients receiving biologics. Physicians who reported not having adjusted the doses to the patients due to the pandemic were 1070 (97.5\%), 1029 (93.8\%), and 704 (64.2\%) for synthetic drugs, biologics, and glucocorticoids, respectively. According to 974 (88.8\%) physicians, patients on antimalarials found difficulties in accessing these drugs during the pandemic.

A change in the administration route for patients' medication was not considered by 917 (83.6\%) rheumatologists, whereas $175(16.0 \%)$ physicians considered a change from intravenous

Table 6. Communication channels used by rheumatologists with their patients aside from the medical consultation $(\mathrm{n}=1097)$.

$\mathrm{n}(\%)$

\begin{tabular}{lc}
\hline Phone call & $600(54.7)$ \\
Text message & $324(29.5)$ \\
Email & $489(44.6)$ \\
WhatsApp & $739(67.4)$ \\
Facebook & $107(9.7)$ \\
Instagram & $44(4)$ \\
Other & $30(2.7)$
\end{tabular}

(IV) to subcutaneous (SC) route for their patients, and 5 (0.5\%) considered a change from SC to IV.

Regarding rheumatologists' participation in the development of COVID-19 local guidelines, 878 respondents (80.0\%) believed they should be involved and 361 (32.9\%) actually participated.

Rheumatologists were asked if they had been required to care for internal medicine patients during the pandemic. An affirmative answer was given by $439(40.0 \%)$ respondents; $192(17.5 \%)$ stated that previously did not care for these kinds of patients, whereas $247(22.5 \%)$ declared that they used to see internal medicine patients. Of those 439 respondents, 177 (40.3\%) stated that they cared for hospitalized patients, 144 (32.8\%) worked with outpatients, 99 (22.6\%) worked with both inpatients and outpatients, and 19 (4.3\%) reported other types of care. A negative answer was given by 658 (60.0\%) respondents.

When those rheumatologists $(n=439)$ were asked if they had been required to care for internal medicine patients with a COVID-19 diagnosis, the question was answered affirmatively by $277(63.1 \%)$ respondents, and of those, $212(76.5 \%)$ stated they had adequate personal protective equipment (PPE).

In addition, rheumatologists were also asked if they had been required to care for rheumatology patients with a COVID-19 diagnosis. This question was answered affirmatively by 338 (30.8\%) respondents, and of those, $265(78.4 \%)$ stated they had adequate PPE.

At least 1 episode of discrimination as a healthcare worker during the pandemic was reported to be experienced by 124 $(11.3 \%)$ of the respondents.

The self-perceived risk of getting infected with SARS-CoV-2 during the pandemic was 50\% (IQR 30-70) and the perceived risk of their family members becoming infected with SARS-CoV-2 during the pandemic was also considered 50\% (IQR 30-70).

\section{DISCUSSION}

Change of medical practice due to the COVID-19 pandemic has been reported worldwide. ${ }^{4-7,20-31}$ Changes in general population behaviors, public health, and medical practice have been reported previously, associated with past outbreaks or pandemics such as Zika, ${ }^{10}$ influenza A (H1N1), ${ }^{11,12,13,15,20}$ SARS, ${ }^{16,17,18,19,26}$ avian influenza $(\mathrm{H} 5 \mathrm{~N} 1),{ }^{14,17,25}$, and chikungunya. ${ }^{27}$

The emergence of COVID-19 led to unprecedented changes to rheumatology clinical practice worldwide, including the restructuring of hospitals and the rapid transition to virtual care. ${ }^{5,21-24,30-32}$ Mehrotra, et al also reported that in a very short time, COVID-19 has promoted a fast conversion from in-person care to telehealth in primary care practices. Changes that would have taken months of planning, pilot testing, and education were performed in 1 or 2 weeks. ${ }^{5}$ In our study, due to COVID-19, in-person practice decreased from 27 hours per week prior to the pandemic, to 10 hours per week during the pandemic.

Rheumatology outpatient departments and hospital services also turned to virtual care during the pandemic. As a norm, patients were recommended not to attend face-to-face visits if they had any symptoms of COVID-19. Adaptations included 
screening for COVID-19 symptoms, mask-wearing, physical distancing in waiting rooms, hand hygiene, and the use of appropriate PPE. ${ }^{1-8,21-23}$

In our sample, in-person care was continued by more than a half of respondents. Similar to our results, in a multinational recent survey that included 554 respondents from 20 countries, face-to-face appointments with the use of personal protective behaviors and equipment continued to be held in $52.9 \%$ rheumatology practices. ${ }^{24}$

The adoption of telehealth channels and methods was acknowledged by $80 \%$ of our respondents. Gkrouzman, et al stated that the COVID-19 outbreak changed the activities of rheumatology services in many ways never seen before. ${ }^{28}$ Nearly all respondents of our survey agreed that the use of telemedicine methods is a valid option during the pandemic, but this percentage declined to $50 \%$ for a hypothetical postpandemic scenario. Considering that the risk of SARS-CoV-2 infection is likely to persist, the integration of telehealth into current models of care will be essential in rheumatology, as in other areas of healthcare. ${ }^{7,8,28,29,30,31}$

The most commonly reported telehealth methods in our study were telephone calls (45.5\%), WhatsApp voice calls (38.6\%), and video calls using platforms such as Skype, WhatsApp, and Microsoft Teams (50.6\%). Similarly, a recent multinational study reported that most common teleconsultation modalities were telephone calls (60.5\%), WhatsApp calls (43.5\%), emails (16.3\%), and video calls (9.6\%). ${ }^{24}$

Barriers to adopting virtual care methods were commonly cited by our respondents. More than $20 \%$ of our sample did not offer telehealth services to their patients. The lack of education or training on remote care methods and the lack of means to offer these alternatives were frequently cited. Video consultation through open and free-access platforms (e.g., Skype, Facebook, or Instagram) were sometimes objected to by healthcare systems, providers, or payers because they do not comply with privacy regulations. ${ }^{24,28,29,30,31,32} \mathrm{~A}$ major reason to avoid offering teleconsultation options was the lack of clarity regarding payment methods.

Nearly half of the respondents said they perceived a reduction in patient adherence to rheumatic drug therapies, with almost no difference between synthetic or biologic agents. In general, rheumatologists did not adjust doses due to the pandemic, and the route of administration was maintained in most cases. Almost $90 \%$ of respondents reported that their patients had some difficulties in accessing antimalarials during the pandemic. According to an international survey conducted by the COVID-19 Global Rheumatology Alliance about antimalarial drug shortages during the pandemic, $6.8 \%$ of patients were unable to continue taking antimalarials because of inadequate supply in the region of the Americas. ${ }^{33}$

The pandemic had a profound economic impact on our respondents. More than $80 \%$ of rheumatologists said they experienced a reduction in monthly wages and the reduction was about $50 \%$ on average. Approximately $50 \%$ of rheumatologists also declared a consultation fee reduction, which on average ended being $40 \%$ less than the usual fee prior to the pandemic. In addition, $8 \%$ of respondents said they had lost their jobs. The negative impact of the pandemic on economics and employment was commonly reported in other studies. ${ }^{28,29}$ According to Keesara, et al, the progress of remote care options requires the growth of adequate payment structures to sustain its development. ${ }^{29}$

In this new COVID-19 scenario, updated practice guidelines will help to improve access to health, reduce costs for patients (e.g., less time away from work, fewer travels), and increase outreach to underserved populations, including those in rural and global communities. ${ }^{3,22,30,31}$ The upcoming new guidelines will certainly reshape rheumatology practice in Latin America. Also, new considerations and regulations about privacy, disclosures, interoperability of electronic health records, and data security will evolve and update as telehealth expands. ${ }^{30,31,32}$ Rheumatologists' involvement in the elaboration of new guidelines was considered essential by $80 \%$ of our respondents and $32 \%$ were already collaborating in these efforts.

Healthcare workers are the first line of defense against COVID-19 and at the same time, they are considered the highest-risk occupational group. According to Betancourt Sanchez, et al, statistics from Italy showed that $20 \%$ of healthcare workers had been infected during the pandemic and in Colombia, the percentage of infected healthcare workers was $7.0 \%{ }^{34}$ In our study, $7.4 \%$ of rheumatologists reported having received a diagnosis of COVID- 19 .

Some estimates suggest that frontline health workers could account for $10-20 \%$ of all COVID-19 diagnoses. ${ }^{35}$ Therefore, it is not surprising that in our study, self-perceived risk of being infected with SARS-CoV-2 was 50\%, with a similar perceived risk for family members.

Compared with healthcare workers who reported adequate availability of PPE, those with inadequate PPE had an increased risk of infection. ${ }^{22,23,24,34,35}$ In our study, nearly $80 \%$ of respondents reported having adequate PPE.

Stigma and discrimination appeared as major issues during the COVID-19 pandemic. Healthcare workers had to face these challenges, including episodes of discrimination in their neighborhoods or workplaces. ${ }^{34,35,36}$ In our study, $11.3 \%$ of respondents experienced at least 1 episode of discrimination as a healthcare worker during the pandemic.

Among the 81 ( $7.4 \%$ of the total sample) rheumatologists who received a diagnosis of COVID-19, 7 (8.6\%) were hospitalized, with a median hospital stay of 11 days. Different medications were administrated to these rheumatologists, hydroxychloroquine and azithromycin being the most frequently reported. At the time we conducted our survey, the results of neither the RECOVERY trial ${ }^{37}$ (dexamethasone) nor the SOLIDARITY trial $^{38}$ (lopinavir/ritonavir, antimalarials, etc.) were available; thus, these medications were not the mainstay treatment in the region.

Drug therapies for COVID-19 reported in our survey are used worldwide. ${ }^{1,3,4,22}$ Globally, the management for SARS-CoV-2 infection was initially extrapolated from previous epidemics of coronaviruses like SARS. ${ }^{16,17,18,19}$ There are no globally approved treatments for COVID-19, and current management of symptomatic patients is based on symptomatic treatment, 
supplemental oxygen, and supportive care. COVID-19 is asymptomatic or minimally symptomatic in more than $80 \%$ of patients and requires no additional management. ${ }^{1,3,21,22}$

Our study has some limitations. The questionnaire was developed de novo, based on the available literature and practice experience. However, it was validated by an independent scientific committee and previously tested for readability, acceptability, and timing in a group of Colombian rheumatologists. The collected data were self-reported by physicians, partly depending on recall ability; this may generate subjectivity and recall bias. Nevertheless, the number of rheumatologists involved from 19 different countries indicates that our study confidently reflects the reality of COVID-19's impact on Latin American rheumatologists.

Given that the survey was distributed by national PANLAR chairs rather than centralized distribution to all PANLAR members, selection bias may be present. Nonetheless, as PANLAR gathers the national rheumatology societies of each of the member countries, and the vast majority of rheumatologists in each country are endorsed by their national society, we consider that only a small number of rheumatologists might have chosen not to answer our survey. Of note, each national PANLAR chair is elected on a democratic basis in their national society.

Our study provided new valuable information about the impact of COVID-19 on rheumatology practice in Latin American countries. Our results described the consequences of the COVID-19 pandemic on rheumatology practice and the professional adaptation to this new scenario. During the pandemic, telehealth has had an important role in healthcare delivery, allowing for ongoing medical care while ensuring the safety of patients and physicians. Careful planning, outcome assessment, and adaptation of existing virtual care methods are future steps needed to achieve a successful integration of telehealth into routine rheumatology practice. In conclusion, the COVID-19 pandemic has reshaped rheumatology practice in Latin America through a wide impact on rheumatologists' clinical practice.

\section{ACKNOWLEDGMENT}

Editorial assistance was provided by Content Ed Net, Madrid, Spain.

\section{REFERENCES}

1. Adhikari SP, Meng S, Wu YJ, Mao YP, Ye RX, Wang QZ, et al. Epidemiology, causes, clinical manifestation and diagnosis, prevention and control of coronavirus disease (COVID-19) during the early outbreak period: a scoping review. Infect Dis Poverty 2020;9:29.

2. Cucinotta D, Vanelli M. WHO declares COVID-19 a pandemic. Acta Biomed 2020;91:157-60.

3. Saavedra Trujillo $\mathrm{CH}$. [Colombian consensus on the care, diagnosis and management of SARS-COV-2/COVID 19 infection in health care facilities. Recommendations based on expert consensus and informed by evidence]. [Article in Spanish] Infectio 2020;24:1-102.

4. Walker PGT, Whittaker C, Watson OJ, Baguelin M, Winskill P, Hamlet A, et al. The impact of COVID-19 and strategies for mitigation and suppression in low- and middle-income countries. Science 2020;369:413-22.
5. Mehrotra A, Ray K, Brockmeyer DM, Barnett ML, Bender JA. Rapidly converting to "virtual practices": outpatient care in the era of Covid-19. NEJM Catalyst 2020;10.1056/CAT.20.0091.

6. Lee TH. Creating the new normal: the clinician response to Covid-19. NEJM Catalyst 2020;10.1056/CAT.20.0076.

7. Figueroa-Parra G, Aguirre-Garcia GM, Gamboa-Alonso CM, Camacho-Ortiz A, Galarza-Delgado DA. Are my patients with rheumatic diseases at higher risk of COVID-19? Ann Rheum Dis 2020;79:839-40.

8. Favalli EG, Ingegnoli F, De Lucia O, Cincinelli G, Cimaz R, Caporali R. COVID-19 infection and rheumatoid arthritis: faraway, so close! Autoimmun Rev 2020;19:102523.

9. Saldarriaga Rivera LM, Fernández Ávila D, Bautista Molano W, Jaramillo Arroyave D, Bautista Ramírez AJ, Díaz Maldonado A, et al. Recommendations on the management of adult patients with rheumatic diseases in the context of SARS-CoV-2/COVID-19 infection. Colombian Association of Rheumatology. Reumatol Clin 2020;16:437-46.

10. Guidry JPD, Carlyle KE, LaRose JG, Perrin P, Messner M, Ryan M. Using the health belief model to analyze Instagram posts about Zika for public health communications. Emerg Infect Dis 2019; 25:179-180.

11. Bults M, Beaujean DJ, Richardus JH, Voeten HA. Perceptions and behavioral responses of the general public during the 2009 influenza A (H1N1) pandemic: a systematic review. Disaster Med Public Health Prep 2015;9:207-19.

12. Lin Y, Huang L, Nie S, Liu Z, Yu H, Yan W. Knowledge, attitudes and practices (KAP) related to the pandemic (H1N1) 2009 among Chinese general population: a telephone survey. BMC Infect Dis 2011;11:128.

13. Ávila J, Munayco CV, Gómez J, Nunura J, Canahuiri J. Knowledge and practices about novel influenza A (H1N1) in health workers and outpatients, Peru (May 2009). Rev Peru Med Exp Salud Pública 2009; 26:328-32.

14. Abbate R, Di Giuseppe G, Marinelli P, Angelillo IF. Knowledge, attitudes, and practices of avian influenza, poultry workers, Italy. Emerg Infect Dis 2006;12:1762-5.

15. Agüero Santagelo F, Nebot Adell M, Pérez Giménez A, López Medina MJ, García Continente X. [Attitudes and preventive behaviours adopted during the (H1N1) 2009 influenza virus epidemic in Spain]. [Article in Spanish] Rev Esp Salud Publica 2011;85:73-80

16. Blendon RJ, Benson JM, DesRoches CM, Raleigh E, TaylorClark K. The public's response to severe acute respiratory syndrome in Toronto and the United States. Clin Infect Dis 2004;38:925-31.

17. Sadique MZ, Edmunds WJ, Smith RD, Meerding WJ, de Zwart O, Brug J, et al. Precautionary behavior in response to perceived threat of pandemic influenza. Emerg Infect Dis 2007;13:1307-13.

18. Lau JT, Yang X, Tsui H, Kim JH. Monitoring community responses to the SARS epidemic in Hong Kong: from day 10 to day 62. J Epidemiol Community Health 2003;57:864-70.

19. Deng JF, Olowokure B, Kaydos-Daniels SC, Chang HJ, Barwick RS, Lee ML, et al; SARS International Field Team. Severe acute respiratory syndrome (SARS): knowledge, attitudes, practices and sources of information among physicians answering a SARS fever hotline service. Public Health 2006;120:15-9.

20. Hashikawa M, Gold KJ. Disaster preparedness in primary care: ready or not? Disaster Med Public Health Prep 2018;12:644-8.

21. Schmulson M, Gudiño-Zayas M, Hani A; Sociedad Latinoamericana de Neurogastroenterología (SLNG). The impact of COVID-19 pandemic on neurogastroenterologists in Latin America: results of an online survey. J Clin Gastroenterol 2020 Sep 2 (E-pub ahead of print). 
22. Mikuls TR, Johnson SR, Fraenkel L, Arasaratnam RJ, Baden LR, Bermas BL, et al. American College of Rheumatology guidance for the management of rheumatic disease in adult patients during the COVID-19 pandemic: version 2. Arthritis Rheumatol 2020;72:e1-12.

23. Cajas Santana LJ, López JG. [Description of a tele-rheumatology service in a Colombian institution]. [Article in Spanish] Rev Colomb Reumatol 2020 Oct 25 (E-pub ahead of print).

24. Akintayo RO, Akpabio AA, Kalla AA, Dey D, Migowa AN, Olaosebikan H, et al. The impact of COVID-19 on rheumatology practice across Africa. Rheumatology 2021;60:392-8.

25. Di Giuseppe G, Abbate R, Albano L, Marinelli P, Angelillo IF. A survey of knowledge, attitudes and practices towards avian influenza in an adult population of Italy. BMC Infect Dis 2008;17;8:36.

26. Rubin GJ, Amlôt R, Page L, Wessely S. Public perceptions, anxiety, and behaviour change in relation to the swine flu outbreak: cross sectional telephone survey. BMJ 2009;339:b2651.

27. Corrin T, Waddell L, Greig J, Young I, Hierlihy C, Mascarenhas M. Risk perceptions, attitudes, and knowledge of chikungunya among the public and health professionals: a systematic review. Trop Med Health 2017;45:21.

28. Gkrouzman E, Wu DD, Jethwa H, Abraham S. Telemedicine in rheumatology at the advent of the COVID-19 pandemic. HSS J 2020;6 Suppl 1:1-4.

29. Keesara S, Jonas A, Schulman K. Covid-19 and health care's digital revolution. N Engl J Med 2020;382:e82.

30. Nune A, Iyengar K, Ahmed A, Sapkota $\mathrm{H}$. Challenges in delivering rheumatology care during COVID-19 pandemic. Clin Rheumatol 2020;39:2817-21.

31. England BR, Barber CEH, Bergman M, RanganathVK, Suter LG, Michaud K. Brief report: Adaptation of American College of
Rheumatology rheumatoid arthritis disease activity and functional status measures for telehealth visits. Arthritis Care Res 2020 Aug 19 (E-pub ahead of print).

32. Temesgen ZM, DeSimone DC, Mahmood M, LibertinCR, VaratharajPalraj BR, Berbari EF. Health care after the COVID-19 pandemic and the influence of telemedicine. Mayo Clin Proc;2020;95:S66-8.

33. Sirotich E, Kennedy K, Surangiwala S, Larche M, Liew J, Wallace Z, et al. Antimalarial drug shortages during the COVID-19 pandemic: results from the Global Rheumatology Alliance patient experience survey [abstract]. Arthritis Rheumatol 2020;72 Suppl 10.

34. Betancourt Sanchez L, Ochoa Gelvez E, Velásquez Bernal C, Rozo Silva IA, Quiroga Vargas DA. [Occupational health in the framework of the COVID-19 pandemic: a scoping review]. [Article in Spanish] Rev Salud Publica 2020;22:1-8.

35. Nguyen LH, Drew DA, Graham MS, JoshiAD, Guo CG, Ma W. Coronavirus Pandemic Epidemiology Consortium. Risk of COVID-19 among front-line health-care workers and the general community: a prospective cohort study. Lancet Public Health 2020;5:e475-83.

36. Roelen K, Ackley C, Boyce P, Farina N, Ripoll S. COVID-19 in LMICs: the need to place stigma front and centre to its response. Eur J Dev Res 2020;21:1-21.

37. Wilkinson E. RECOVERY trial: the UK covid-19 study resetting expectations for clinical trials. BMJ 2020;369:m1626

38. World Health Organization. "Solidarity" clinical trial for COVID-19 treatments [Internet. Accessed June 2, 2021.] Available from: www.who.int/emergencies/diseases/novel-coronavirus-2019/ global-research-on-novel-coronavirus-2019-ncov/ solidarity-clinical-trial-for-covid-19-treatments 